\title{
Conductance of quantum spin Hall edge states from first principles: The critical role of
} magnetic impurities and inter-edge scattering

\author{
Vannucci, Luca; Olsen, Thomas; Thygesen, Kristian Sommer
}

Published in:

Physical Review B

Link to article, DOI:

10.1103/physrevb.101.155404

Publication date:

2020

Document Version

Publisher's PDF, also known as Version of record

Link back to DTU Orbit

Citation (APA):

Vannucci, L., Olsen, T., \& Thygesen, K. S. (2020). Conductance of quantum spin Hall edge states from first principles: The critical role of magnetic impurities and inter-edge scattering. Physical Review B, 101(15), [155404]. https://doi.org/10.1103/physrevb.101.155404

\section{General rights}

Copyright and moral rights for the publications made accessible in the public portal are retained by the authors and/or other copyright owners and it is a condition of accessing publications that users recognise and abide by the legal requirements associated with these rights.

- Users may download and print one copy of any publication from the public portal for the purpose of private study or research.

- You may not further distribute the material or use it for any profit-making activity or commercial gain

- You may freely distribute the URL identifying the publication in the public portal 


\title{
Conductance of quantum spin Hall edge states from first principles: The critical role of magnetic impurities and inter-edge scattering
}

\author{
Luca Vannucci, ${ }^{1, *}$ Thomas Olsen, ${ }^{1}$ and Kristian S. Thygesen ${ }^{1,2}$ \\ ${ }^{1}$ CAMD, Department of Physics, Technical University of Denmark, 2800 Kongens Lyngby, Denmark \\ ${ }^{2}$ Center for Nanostructured Graphene (CNG), Technical University of Denmark, 2800 Kongens Lyngby, Denmark
}

(Received 10 January 2020; revised manuscript received 27 February 2020; accepted 10 March 2020;

published 2 April 2020)

\begin{abstract}
The outstanding transport properties expected at the edge of two-dimensional time-reversal invariant topological insulators have proven to be challenging to realize experimentally, and have so far only been demonstrated in very short devices. In search for an explanation to this puzzling observation, here we report a full first-principles calculation of topologically protected transport at the edge of novel quantum spin Hall insulators-specifically, bismuth and antimony halides-based on the nonequilibrium Green's functions formalism. Our calculations unravel two different scattering mechanisms that may affect two-dimensional topological insulators, namely, time-reversal symmetry breaking at vacancy defects and inter-edge scattering mediated by multiple cooperating impurities, possibly nonmagnetic. We discuss their drastic consequences for typical nonlocal transport measurements as well as strategies to mitigate their negative impact. Finally, we provide an instructive comparison of the transport properties of topologically protected edge states to those of the trivial edge states in $\mathrm{MoS}_{2}$ ribbons. Although we focus on a few specific cases (in terms of materials and defect types), our results should be representative for the general case and thus have significance beyond the systems studied here.
\end{abstract}

DOI: 10.1103/PhysRevB.101.155404

\section{INTRODUCTION}

In a pair of groundbreaking papers published in 2005, Kane and Mele argued that graphene becomes a two-dimensional topological insulator (2D TI) at sufficiently low temperature $[1,2]$. In other words, a flake of graphene cooled down to cryogenic temperature will essentially behave as a band insulator everywhere except on its boundaries, where special metallic edge states will appear. Such states are immune to scattering against impurities or disorder, and therefore realize a perfect dissipationless conductor with great potential for future technological applications. The reason behind this striking robustness is rooted in the mathematical concept of topology, hence the name topological insulators [3].

Unfortunately, the band gap opened by spin-orbit coupling (SOC) in graphene is actually too small to give rise to any measurable effect [4]. To realize the first 2D TI-or quantum spin Hall insulator (QSHI) - it was therefore necessary to resort to quantum-well heterostructures, which indeed showed the much anticipated signatures of topologically protected transport in nonlocal multiterminal measurements $[5,6]$. With the rising awareness that the realm of monolayer 2D materials is actually much larger than initially thought, several new QSHIs have been reported in other 2D monolayers than graphene in recent years [7-10].

It is, however, not fully understood to what extent the ideas of topological protection can materialize into the next generation of electronic devices, due to some inconsistency between theory and experiments. Time-reversal symmetry (TRS) forbids electron backscattering on the edge of $2 \mathrm{D}$ TIs, since

*lucav@fysik.dtu.dk counterpropagating modes have opposite spin polarizationthey are therefore termed helical edge states. The defining feature of such a pair of protected states is a well-defined quantized conductance plateau at $G_{0}=2 e^{2} / h$, which is, however, difficult to attain in the laboratory. The few successful attempts are all limited to very low temperature $(\sim 1 \mathrm{~K}$ in quantum-well heterostructures $[5,6])$ or very short channels $\left(\sim 100 \mathrm{~nm}\right.$ for the case of monolayer $\mathrm{WTe}_{2}$ [8]). Attempts to understand this discrepancy at the model level have focused on many diverse backscattering mechanisms driven by electron-electron interactions, charge puddles, embedded nuclear spins, coupling to phonons, and electromagnetic noise [11-23]. Nonetheless, the question is still much debated and deserves a careful analysis from a different and more realistic point of view.

Here we report a full first-principles study of topologically protected transport at the edge of novel QSHIs. We use recently developed computational 2D materials databases [24,25], containing existing structures as well as hitherto unknown monolayers, to identify a family of large-gap QSHIs ideally suited for the current study. We then explore the electronic band structure of such candidates at the level of density functional theory (DFT), both as infinite bulk monolayers and in different nanoribbon geometries. For nanoribbons, we highlight the emergence of robust metallic states whose eigenvalues cross the region of the bulk gap, and investigate the robustness of their transport properties in the framework of the nonequilibrium Green's functions (NEGF) formalism [26], with full account of spin-orbit interactions. Our calculations show that naturally occurring native defects at the edge can spontaneously acquire a magnetic moment, thereby violating TRS and leading to a suppression of edge transport. This 
result reveals the mechanism that is most likely to affect edge conduction in 2D topological insulators. Interestingly, we find that chemical saturation of vacancy defects (e.g., by hydrogen) is sufficient to remove the magnetic moment, thereby providing a strategy to restore topological protection at the edge. Perhaps more surprisingly, even nonmagnetic impurities may be detrimental for transport. We indeed show that multiple nonmagnetic impurities may create a channel for interedge scattering between opposite edge states in relatively wide ribbons, thereby affecting transport properties even when they do not represent a threat to transport individually.

The structure of this paper is as follows. In Sec. II, we illustrate the family of topological materials studied in this work and present their band structure in the zigzag nanoribbon geometry. In the subsequent Secs. III and IV, we calculate the transport properties of topological nanoribbons in the presence of impurities, and discuss the defect-induced intra-edge and inter-edge backscattering mechanisms. A similar formalism is then applied to zigzag $\mathrm{MoS}_{2}$ nanoribbons in Sec. V, thereby providing an insightful comparison with the transport properties of topologically trivial edge states. Finally, Sec. VI summarizes the main results of this paper. The Appendix is dedicated to technical details about the DFT calculations.

\section{ELECTRONIC STRUCTURE OF TOPOLOGICAL NANORIBBONS}

The aim of this work is to address the problem of topologically protected transport by going beyond the simple model approximation, and to perform full transport calculations of realistic topological compounds from first principles. Thanks to the application of automated high-throughput methods in the context of material science, the portfolio of theoretically proposed 2D materials is nowadays expanding at a remarkable pace $[24,25]$. This has led to the identification of several new candidates for 2D topological insulators [27-29], some of which are now being tested in the laboratory $[9,10,30]$.

Here we choose to focus on bismuth and antimony halides, i.e., binary compounds $\mathrm{Bi} X$ and $\mathrm{Sb} X$, with $X=(\mathrm{F}, \mathrm{Cl}, \mathrm{Br}$, I), whose topological nature has already been investigated in earlier works $[28,31,32]$. The reason for our choice is threefold:

(i) They are simple binary compounds of $\mathrm{Bi} / \mathrm{Sb}$ and a halogen element, with a rather small number of valence electrons per unit cell. This allows us to deal with bigger devices without extreme computational effort (compared to other candidates).

(ii) They are dynamically stable and thermodynamically metastable, in the sense that their heat of formation is negative with respect to the pure elemental form and not much larger than other competing phases (see Ref. [25] for further discussion about thermodynamic stability of $2 \mathrm{D}$ materials). This means that the compound might be synthesizable, although this is not an essential aspect of our work. Indeed, we expect our conclusions to be universally applicable to any QSHI.

(iii) Their band gap is predicted to be in the range 0.4 $1.0 \mathrm{eV}$, which is significantly larger than most other candidates and allows for an easy distinction between topologically protected transport mediated by in-gap edge states and trivial bulk transport.
Monolayer bismuth (antimony) halides consist of a hexagonal lattice of $\mathrm{Bi}(\mathrm{Sb})$ atoms sandwiched between two layers of halogen atoms (see Fig. 1). Halide atoms are disposed above and below the central layer in an alternate fashion, so that each bismuth (antimony) is bonded to one halogen only - a similar structure occurs for the so-called graphane $[33,34]$. Lattice parameters and band structures for infinite 2D monolayer are all reported in the Supplemental Material [35].

Since they are band insulators with nontrivial topological invariant $\mathbb{Z}_{2}=1[28,31]$, the interface between BiX/SbX and a trivial insulator (e.g., vacuum) should host a pair of helical edge states. We confirm this by investigating the electronic structure of zigzag-terminated ribbons of different widths, such as the one shown in Fig. 1. Since materials with the same group-15 element but different halogens behave in a qualitatively similar fashion, we will focus on $\mathrm{BiBr}$ and $\mathrm{SbBr}$, and show results for the remaining materials in the Supplemental Material [35].

Figure 1 shows the band structure of $\mathrm{BiBr}$ and $\mathrm{SbBr}$ zigzag nanoribbons across half of the 1D Brillouin zone. In stark contrast with infinite 2D structures, all nanoribbons are gapless and show robust metallic states lying in the region of the bulk gap, which is of the order of $0.8 \mathrm{eV}$ for $\mathrm{BiBr}$ and $0.4 \mathrm{eV}$ for $\mathrm{SbBr}$. Such states, highlighted in green in Fig. 1, are remarkably stable as the width of the ribbon is increased, as opposed to the remaining valence and conduction states which become more and more dense and are displaced in energy as we move towards larger ribbons. We thus conclude that metallic in-gap states are localized on the edge, a fact which is also confirmed by inspecting the corresponding wave function (see Fig. S4 in the Supplemental Material [35]). Note that the spectrum is spin degenerate owing to the presence of inversion symmetry, but metallic bands with opposite spin are located on opposite edges.

It is worth noticing that the presence of edge states is attributed to the nontrivial topological character of the bulk bands, and not to the particular edge termination. Indeed, we observe qualitatively similar features in both zigzag and armchair nanoribbons, with the latter reported in the Supplemental Material for completeness [35].

\section{BREAKDOWN OF EDGE TRANSMISSION DUE TO MAGNETIC EDGE DEFECTS}

To calculate the edge transport properties of bismuth and antimony halides, we make use of the software package QUANTUMATK [36,37], which allows one to simulate transport devices in the framework of the NEGF formalism [26]. We will focus on the zero-bias transmission spectrum (TS) of a two-terminal device over an energy window of $3 \mathrm{eV}$ that includes the bulk gap. This is straightforwardly linked to the two-terminal conductance of a real device through the well-known Landauer formula $G=\frac{e^{2}}{h} T\left(E_{\mathrm{F}}\right)$ [38].

Our setup is illustrated in Fig. 2 and is made as follows. For any given material, we create two identical, semi-infinite, pristine electrodes by repeating the nanoribbon unit cell shown in Fig. 1 . We then create a central scattering region by considering a finite-length portion of TI nanoribbon, and remove one or more halogen atoms to account for the presence of vacancy defects (denoted as $V_{X}$, with $X$ being the missing atom). 

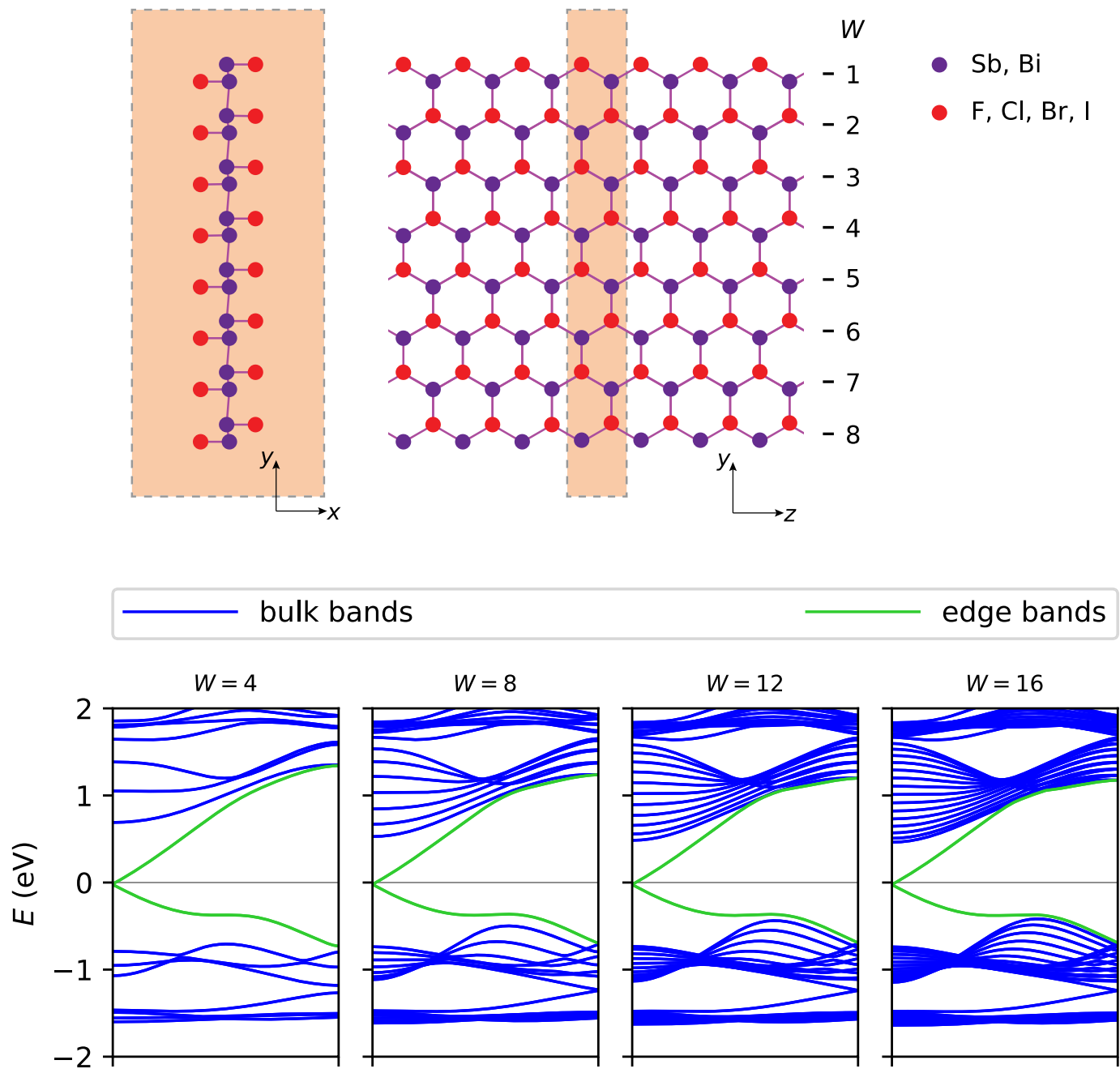

$\mathrm{BiBr}$
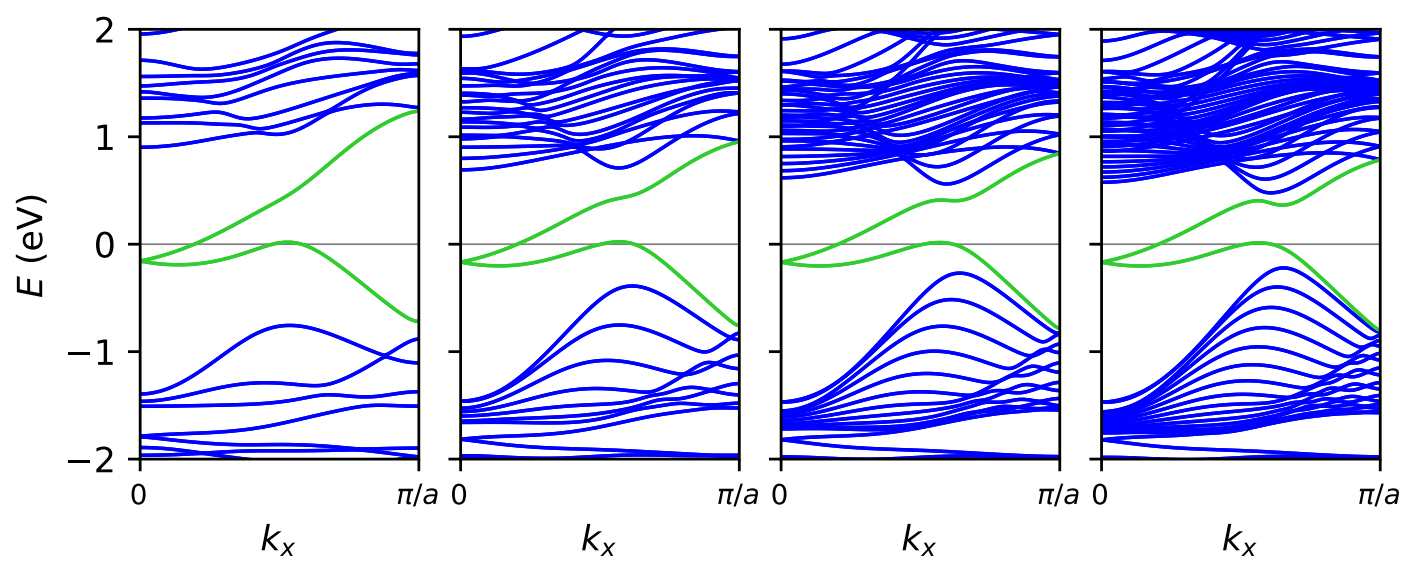

$\mathrm{SbBr}$

FIG. 1. Top: A bismuth or antimony halide zigzag nanoribbon of width $W=8$. Dark purple dots denote the positions of Bi or Sb atoms, while red dots denote the halogen atoms $(\mathrm{F}, \mathrm{Cl}, \mathrm{Br}$, or I). The shaded region corresponds to the unit cell used to represent the infinite ribbon. Bottom: Band structure of $\mathrm{BiBr}$ and $\mathrm{SbBr}$ zigzag nanoribbons of different width $W$. Topological edge states are highlighted in green. Energy is measured with respect to the Fermi energy.

By connecting together the left electrode, central scattering region, and right electrode, we obtain a two-terminal device setup for transport calculations.

Let us first focus on the case of a halogen vacancy on the edge (hereafter named edge defect), as shown in Fig. 2. Transmission spectra for $W=8$ zigzag nanoribbons with edge defects are reported with red dashed lines in Fig. 2, where we also report the band structure and TS of pristine ribbons.

As shown in Fig. 2(b), introducing an edge defect in $\mathrm{BiBr}$ does not lead to any observable effect in the region of the edge states' dispersion, where the transmission exactly 

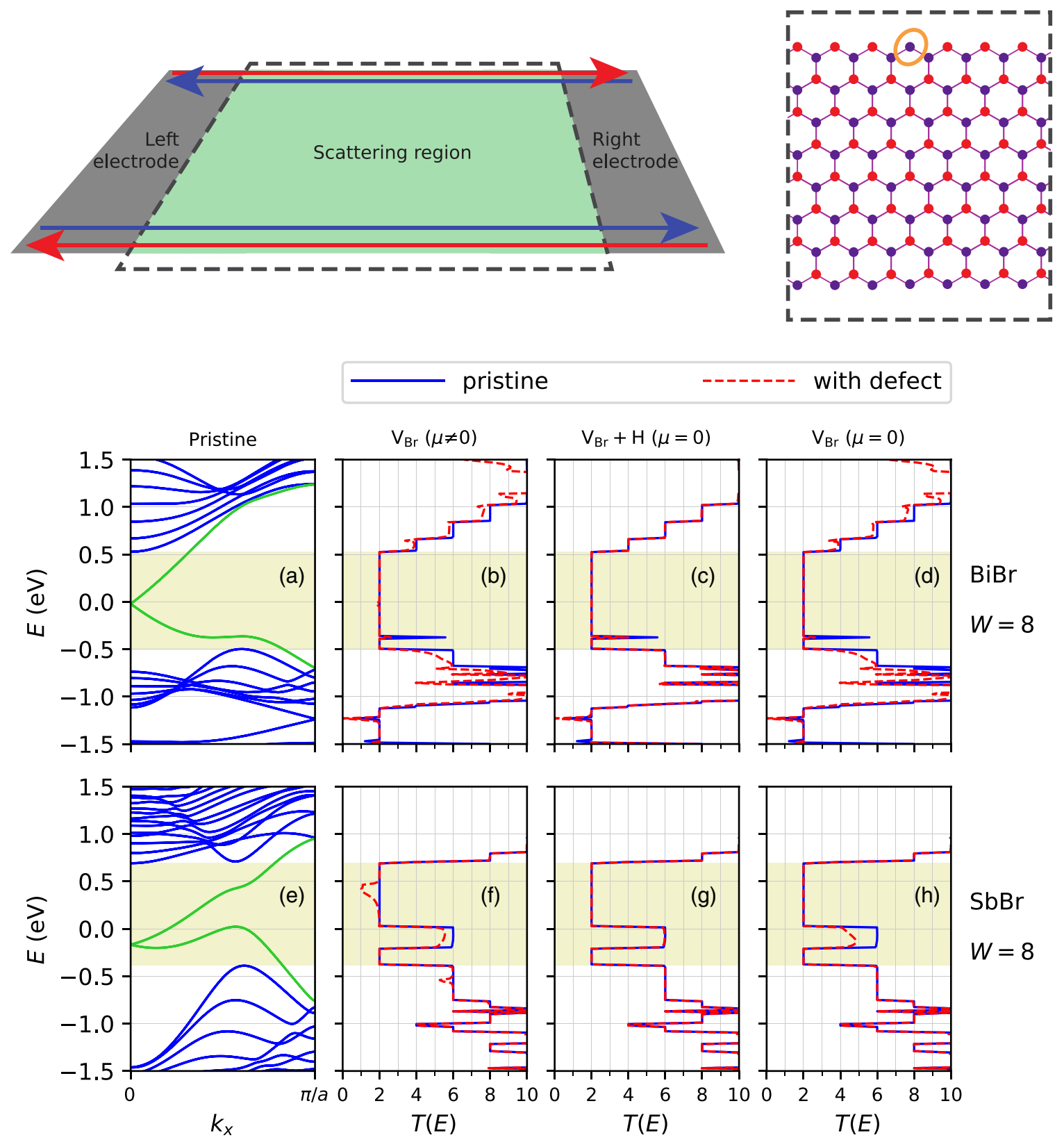

FIG. 2. Top: Sketch of the setup used for transport calculations. Two semi-infinite electrodes (in gray) are connected to the scattering region in the middle (light green). The latter is a finite-size nanoribbon with one or more vacancy defects. A central region with an edge defect is shown to the right. Bottom: Transmission spectrum (TS) of $\mathrm{BiBr}$ and $\mathrm{SbBr}$ zigzag nanoribbons of width $W=8$ in the presence of one of the following edge defects: (b),(f) magnetic edge defect; (c),(g) hydrogen-saturated edge defect; (d),(h) nonmagnetic edge defect. The TS for a pristine ribbon is also shown for comparison in each panel, and its band structure is reported in (a) and (e) from Fig. 1. The energy region of insulating bulk is highlighted in yellow.

equals the number of bands. On the other hand, it does give a partial suppression of transmission for bulk valence and conduction states. Such a behavior is indeed the hallmark of 2D TIs, whose edge states' conduction is protected even in the presence of disorder as long as time-reversal symmetry is not broken. However, the same consideration does not hold for the case of $\mathrm{SbBr}$ [see Fig. 2(f)], which shows an unexpected and well-pronounced antiresonance in the TS around $0.4 \mathrm{eV}$ and therefore a failure of topological protection. A similar feature, although much less pronounced, can be also observed around $-0.5 \mathrm{eV}$.
To investigate the origin of the transmission dip, we have checked the distribution of magnetic moments for the entire device. It shows that the configuration is almost entirely nonmagnetic, except for a magnetic moment of $0.9 \mu_{\mathrm{B}}$ which is exactly localized at the $\mathrm{Sb}$ site underneath the $\mathrm{Br}$ vacancy. On the other hand, the $\mathrm{BiBr}$ nanoribbon has a negligible small magnetic moment at the Br vacancy. We thus attribute the suppression of edge conductance in $\mathrm{SbBr}$ nanoribbons to the spontaneous magnetization of the edge impurity, which invalidates topological protection and allows for intra-edge backscattering. This is a recurrent feature for all antimony 
halides, for which we systematically observe an antiresonance in a narrow energy window around $0.2-0.5 \mathrm{eV}$ due to the formation of localized magnetic moments (see Supplemental Material [35]).

It is interesting to notice that a similar mechanism has been proposed very recently in the framework of the KaneMele-Hubbard model in graphene, where the breakdown of time-reversal symmetry at vacancy defects is shown to lead to a corresponding breakdown of conductance quantization [23]. The present $a b$ initio calculations support this picture and also bear similarities with simple theoretical models in which magnetic scatterers, such as magnetic adatoms or ferromagnetic gates, are introduced in 2D TIs [39,40].

The magnetization of edge defects originates from the presence of a dangling bond at the vacancy site, which makes the configuration chemically unstable and drives the formation of a localized magnetic moment, as reported in earlier work [41-46]. We thus conjecture that the chemical saturation of the dangling bond with a suitable element should eliminate any magnetic structure at the defect and restore a perfect transport at the edge. Indeed, we find that saturation with hydrogen restores the perfect transmission, as shown in Figs. 2(c) and 2(g).

We have also calculated the TS for a nonmagnetic configuration of the halogen vacancy (we manually set all magnetic moments to zero), which we report in Figs. 2(d) and 2(h). As expected, there is no backscattering in this case since time-reversal symmetry is not violated. However, we find a rather small energy difference between the magnetic and nonmagnetic configurations, which might be beyond the accuracy of DFT calculations.

There is, however, one last puzzling question emerging from Fig. 2, which is the unexpected backscattering observed in $\mathrm{SbBr}$ nanoribbons between -0.2 and $0.0 \mathrm{eV}$, that is, where $T(E)=6$ [see Figs. 2(f) and 2(h)]. We attribute this result to the following mechanism. Due to the presence of nonmonotone energy dispersion of the edge states, the nanoribbon actually hosts three pairs of metallic states per edge in this energy range, whose direction of motion can be easily inferred from the slope of the bands. ${ }^{1}$ Thus, it becomes possible for an electron to scatter into a state with the same spin but different direction of motion, as schematically depicted in Fig. 3. This mechanism can never lead to the total suppression of edge conductance, as there will always be a pair of helical edge states which are not accompanied by the corresponding counterpropagating states. In other words, in the presence of impurities or disorder, whatever odd number of edge states' pairs is practically equivalent to a single pair, which is a manifestation of the binary nature of the $\mathbb{Z}_{2}$ topological invariant. This implies that the TS in the region between -0.2 and $0.0 \mathrm{eV}$ may approach $T(E)=4$ as the impurities become more and more abundant, since the three transport channels on the bottom edge will be accompanied by only one surviving pair on the disordered top edge. To check this, we have investigated different nonmagnetic configurations of $\mathrm{SbBr}$ nanoribbons with multiple edge defects, which are all

\footnotetext{
${ }^{1} \mathrm{~A}$ similar effect, although much less pronounced, occurs for $\mathrm{BiBr}$ around $-0.4 \mathrm{eV}$.
}

reported in Fig. 3 together with the single-impurity configuration previously discussed. Indeed, the resulting TS never drops below 4 in the aforementioned region, supporting our interpretation.

In passing, it is worth noting that we have also calculated the TS for smaller ribbons $(W=4)$ and for all remaining materials in the presence of similar edge defects, obtaining qualitatively similar results - that is, sharp antiresonance in the conductance due to the formation of magnetic defects, and intra-edge backscattering when the structure carries three helical pairs per boundary. These results are shown in the Supplemental Material [35].

\section{INTER-EDGE SCATTERING MEDIATED BY NONMAGNETIC BULK DEFECTS}

We now turn our attention to halogen vacancies located away from the edge, which will be denoted bulk defects. As for the case of edge defects, we have studied both magnetic and nonmagnetic configurations. However, we will only present results for nonmagnetic bulk defects in $\mathrm{BiBr}$ for the sake of clarity.

We have explored a scenario where two halogen atoms are simultaneously removed from a $\mathrm{BiBr}$ nanoribbon of width $W=8$, leaving a couple of bulk vacancy defects in the central region. As shown in Fig. 4, both vacancies are symmetrically placed at a distance $d=\sqrt{3} a$ from the edge, with the defectdefect distance being $d^{\prime}=5 a / \sqrt{3}$ (i.e., $d=9.46 \AA$ and $d^{\prime}=$ $15.76 \AA$ for $\mathrm{BiBr}$ ).

The TS of such a configuration is reported in Fig. 4. While both impurities do not affect transport properties in the bulk gap region individually, which is demonstrated by the perfect TS in the region $-0.5 \lesssim E \lesssim 0.5 \mathrm{eV}$ in Fig. 4(b), they do suppress transport at energy $E \approx 0$ when they are simultaneously present, which is reflected in the sharp antiresonance in Fig. 4(c).

The corresponding scattering event is of inter-edge nature, as intra-edge scattering is forbidden by TRS (we are considering a nonmagnetic structure). An electron traveling along the top boundary from left to right can hop on the closest defect state, whose energy actually lies in the bulk gap range. From there, it has a finite possibility of reaching the second impurity, due to a nonzero matrix element between localized states at the impurities. Finally, it tunnels into the bottom edge states, where it propagates back towards the left electrode without having to flip the spin polarization. This analysis is further confirmed by the behavior of the local density of states shown in Fig. 5, which clearly demonstrates that the impurity states occupy a large transverse portion of the nanoribbon, while also having a substantial overlap between them. In this scenario, it is the cooperation between multiple impurities that creates a path for inter-edge scattering, even in such cases where they would not represent any threat to transport if considered individually. Such a mechanism has been frequently neglected in the literature, which rather focuses on the effect of single impurities on the transport properties of $2 \mathrm{D}$ TIs. Nevertheless, it could play a major role when the defect concentration exceeds a certain threshold $[47,48]$ or when opposite edge states are deliberately funneled through a narrow constriction [49]. 

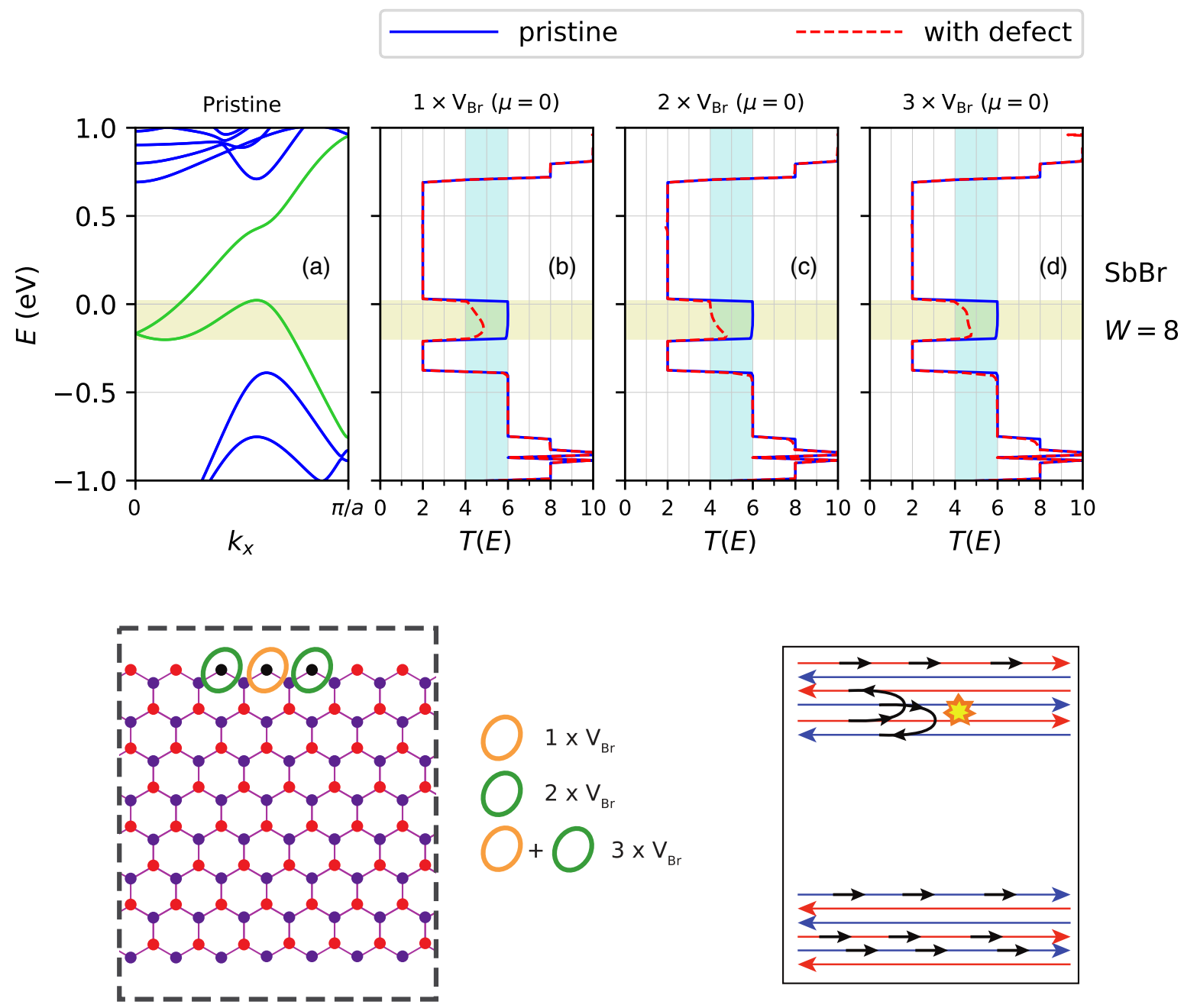

FIG. 3. Transmission spectrum (TS) of an SbBr zigzag nanoribbon of width $W=8$ in the presence of multiple nonmagnetic edge defects as shown in the bottom panel: (b) single-edge defect, reported from Fig. 2(h); (c) double-edge defect; (d) triple-edge defect. The TS for a pristine ribbon is also shown for comparison in each panel, and its band structure is reported from Fig. 1 in (a). The energy region in which the nanoribbon bears three pairs of edge states in highlighted in yellow, while the region $4 \leqslant T(E) \leqslant 6$ is highlighted in cyan. A graphical interpretation of the results in terms of open and closed channels on each edge is given in the bottom right panel, where metallic edge states with opposite spin polarization are represented with different colors (red and blue).

It is worth noting that chemical saturation of the dangling bonds with hydrogen removes the energy levels of the defects from the bulk gap region. The corresponding TS, which we show in Fig. 4(d), is basically unaffected, thus suggesting a strategy to minimize the impact of impurity-mediated interedge scattering on transport.

Finally, we mention that we have observed signatures of inter-edge scattering for different disordered configurations and nanoribbon widths, as reported in the Supplemental Material [35]. In particular, opposite edge states in narrow nanoribbons $(W=4)$ can be coupled together by one single bulk impurity, since the spatial extension of the defect wave function becomes comparable with the nanoribbon width [35].

\section{COMPARISON WITH NONTOPOLOGICAL EDGE STATES}

So far, we have discussed two mechanisms that lead to the failure of conductance quantization in 2D TIs. In this section, we illustrate how the results presented up to now are clearly related to topology, and how edge states would behave in the absence of topological protection.

We thus focus here on the transport properties of a $\mathrm{MoS}_{2}$ nanoribbon, whose crystal structure is shown in Fig. 6. Such structures are known to host metallic edge states at the zigzag termination which are, however, not due to topology, but rather to polar discontinuity at the interface between $\mathrm{MoS}_{2}$ and the vacuum [50,51]. We will therefore call these trivial edge states, to indicate that they are not generated as boundary states between materials with different topological invariants.

In Fig. 6(a), we show the band structure of a pristine $\mathrm{MoS}_{2}$ nanoribbon of width $W=8$. The metallic edge states, indicated with a different color in the figure, are clearly visible. We then create two different defect configurations by removing an atom on the edge (either Mo or S). We calculate the TS for such structures neglecting the contribution from SOC, which is known to have a negligible effect in this case, and compare them to the TS of a pristine nanoribbon. 

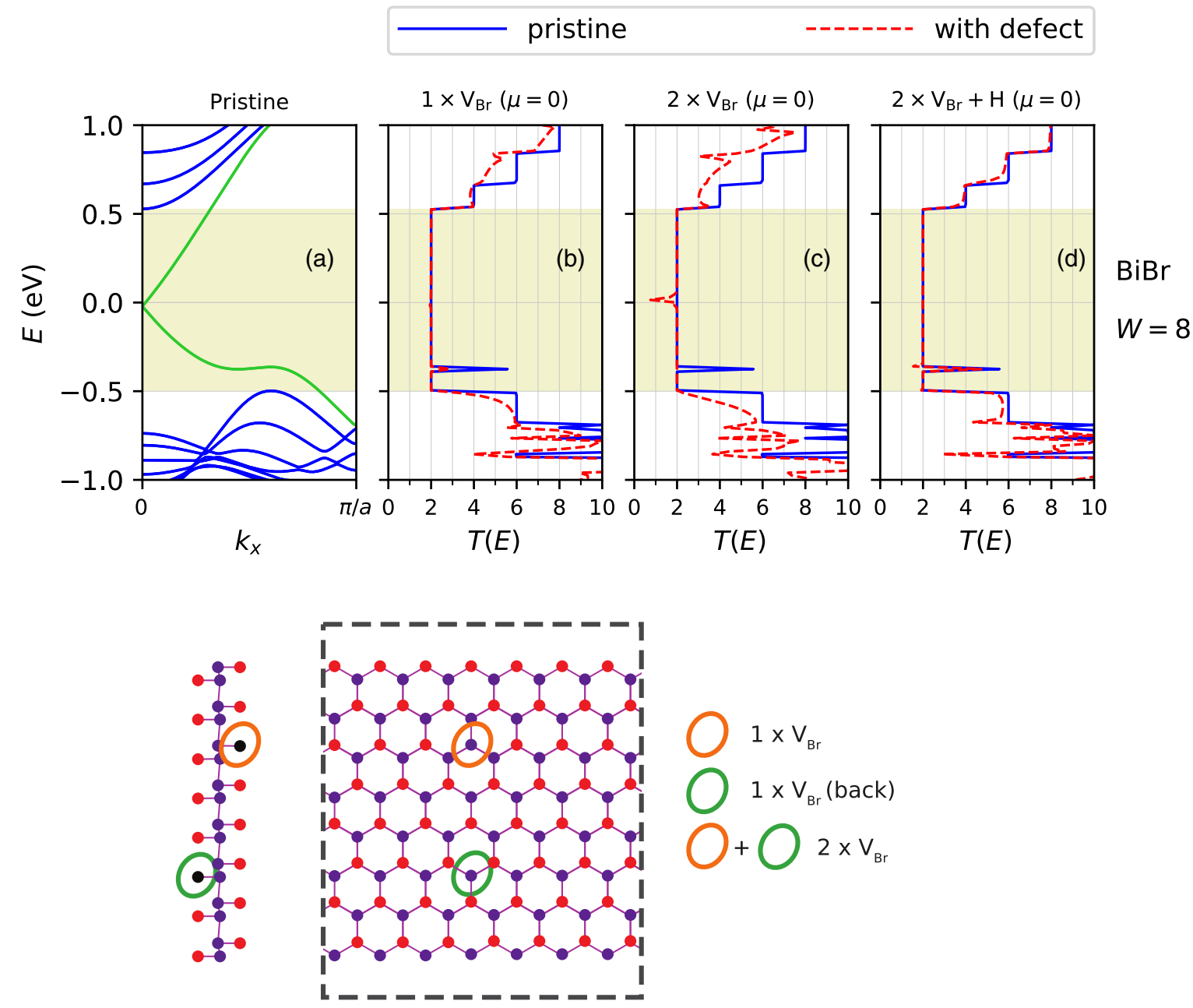

FIG. 4. Transmission spectrum (TS) of a BiBr zigzag nanoribbon of width $W=8$ in the presence of multiple nonmagnetic bulk defects as sketched in the bottom panel: (b) single bulk defect; (c) double bulk defect; (d) hydrogen-saturated double bulk defect. The TS for a pristine ribbon is also shown for comparison in each panel, and its band structure is reported in (a) from Fig. 1. The energy region of insulating bulk is highlighted in yellow.

(a)

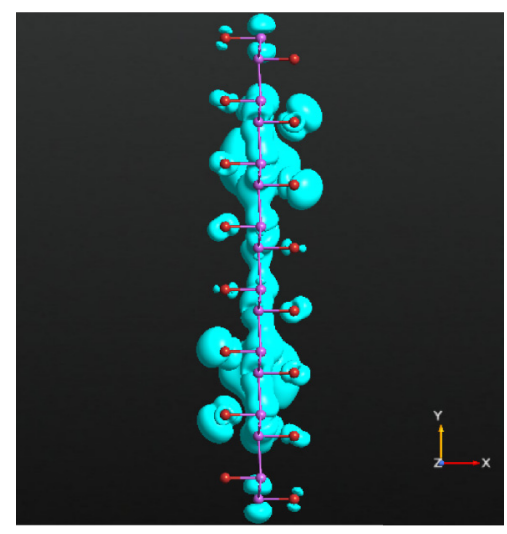

(b)

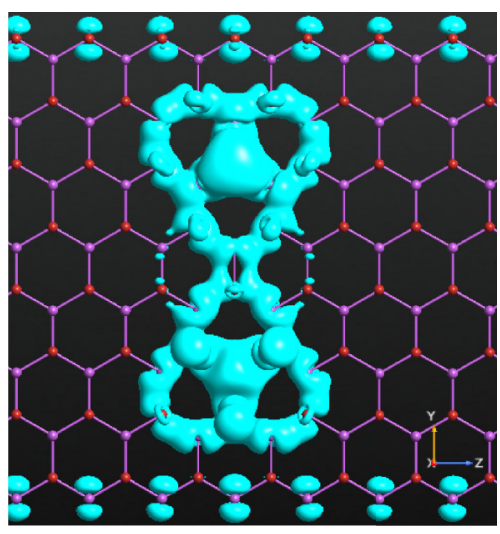

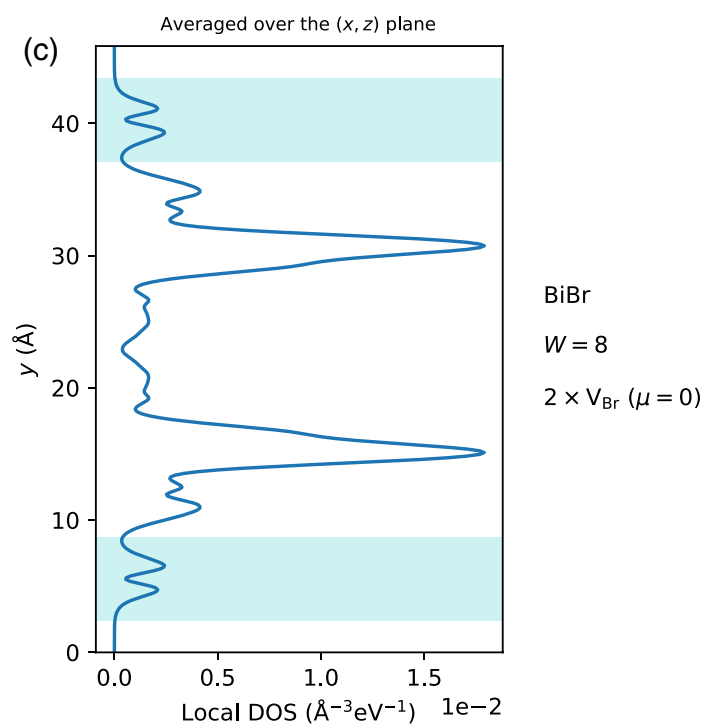

FIG. 5. Local density of states (LDOS) for the configuration in Fig. 4. (a) Side and (b) top views of the surface LDOS( $x, y, z)=$ $0.02 \AA^{-3} \mathrm{eV}^{-1}$. (c) The LDOS in the transverse direction $y$ averaged over the $x z$ plane, with the spatial region spanned by the edge states highlighted in cyan. 

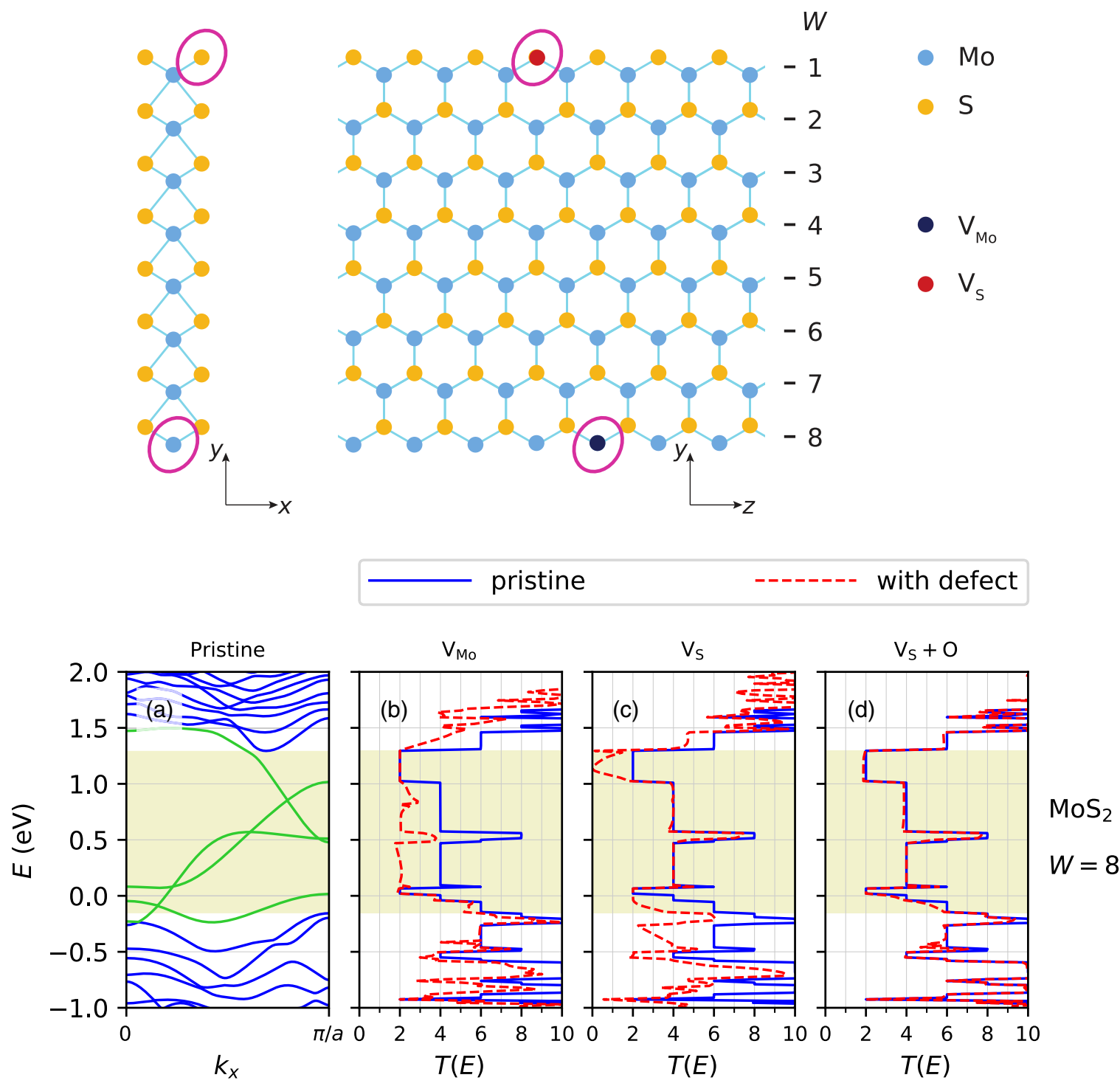

FIG. 6. Top: Top and lateral view of an $\mathrm{MoS}_{2}$ zigzag nanoribbon of width $W=8$. Atoms removed to create defects are denoted with different colors. Bottom: Transmission spectrum (TS) of MoS2 zigzag nanoribbons of width $W=8$ in the presence of one of the following edge defects shown in Fig. 6: (b) Mo vacancy; (c) S vacancy; (d) oxygen-saturated S vacancy. The TS for a pristine ribbon is also shown for comparison in each panel, and its band structure is shown in (a). The energy region of insulating bulk is highlighted in yellow.

Figures 6(b) and 6(c) show that the presence of an edge defect has a dramatic consequence for the transmission properties of the nanoribbon. Generally, we observe a much larger effect of backscattering as compared to the case of topologically protected materials. The conductance is basically halved over the entire energy range $0.0-1.0 \mathrm{eV}$ when we remove a single Mo atom from the edge, and is completely blocked in the range $1.0-1.3 \mathrm{eV}$ when the edge defect is a sulfur vacancy. We conclude that the trivial edge states of $\mathrm{MoS}_{2}$ nanoribbons are much more prone to backscattering than the topologically protected edge states in the $\mathrm{Bi}(\mathrm{Sb})$ halides.

Finally, we have also calculated the TS for the case of oxygen-saturated $\mathrm{S}$ vacancy, which is shown in Fig. 6(d). Once again, chemical saturation seems to be beneficial to edge transport, as we recover an almost perfect transmission. However, the TS still deviates by $3 \%$ from the pristine value in the region $0.5-1.3 \mathrm{eV}$. In contrast, the TS for $\mathrm{H}$-saturated edge defects in $\mathrm{BiBr}$ and $\mathrm{SbBr}$ nanoribbons never deviates more that $0.1 \%$ from the corresponding quantized values.

\section{CONCLUSIONS}

In summary, we have performed ab initio transport calculations of two-terminal TI nanoribbons using the NEGF formalism. By accounting for the presence of both edge and bulk defects, we have pinpointed two sources of backscattering which lead to the breakdown of conductance quantization:

(i) Intra-edge scattering due magnetic edge impurities. The dangling bond originated by the removal of one atom at the edge may in some cases drive the formation of a localized magnetic moment at the impurity site. This local breaking of TRS allows for backscattering events involving spin flip [as sketched in Fig. 7(a)]. This basically blocks electrical conduction through one of the edges, while leaving the opposite 

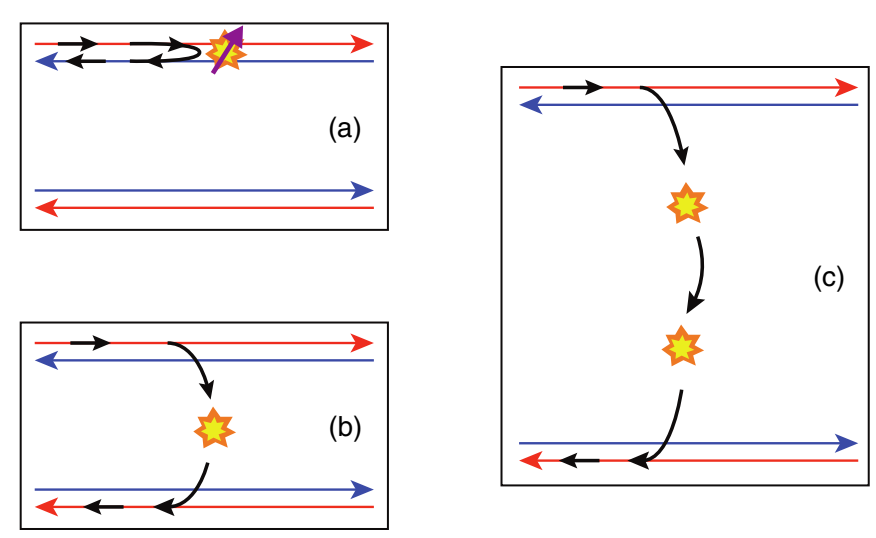

FIG. 7. Graphical representation of different backscattering mechanisms: (a) intra-edge scattering due to magnetic edge impurities, (b) inter-edge scattering through a single nonmagnetic bulk defect, (c) inter-edge scattering mediated by multiple bulk impurities. Metallic edge states with opposite spin polarization are represented with different colors (red and blue).

one unperturbed. The corresponding transmission function drops from 2 to 1 when the energy of the incoming electron resonates with the defect level.

(ii) Inter-edge scattering due to (multiple) bulk impurities. In a narrow nanoribbon, a single nonmagnetic bulk impurity can open a backscattering channel between opposite edge states without breaking TRS [Fig. 7(b)]. Although this can obviously be avoided by increasing the nanoribbon width, large nanoribbons will still be affected by inter-edge scattering above a certain threshold of defect concentration, when multiple bulk impurities generate a backscattering path across the structure [Fig. 7(c)].

Finally, it is worth mentioning that our results are by no means limited to the particular class of materials chosen in this work. Rather, we expect them to be relevant for all QSHIs. We also anticipate that similar mechanisms may deteriorate surface conduction of three-dimensional time-reversal invariant TIs.

\section{ACKNOWLEDGMENTS}

We thank Nicola Marzari for stimulating discussions, and Mads Brandbyge and Tue Gunst for useful discussions and technical help. The research leading to these results has received funding from the European Union's Horizon 2020 research and innovation program under the Marie Skłodowska-Curie Grant Agreement No. 754462. (EuroTechPostdoc). K.S.T. acknowledges funding from the European Research Council (ERC) under the European Union's Horizon 2020 research and innovation programme (Grant No. 773122, LIMA). The Center for Nanostructured Graphene is sponsored by the Danish National Research Foundation, Project No. DNRF103.

\section{APPENDIX: METHODS}

All structures are obtained directly from the C2DB database [25], where they are relaxed with the Perdew-BurkeErnzerhof (PBE) [52] exchange-correlation functional using the DFT code GPAW $[53,54]$ and the software package ASE [55]. For further details about this first step, we refer the reader to Ref. [25].

To create a nanoribbon, we define a rectangular unit cell as shown in Fig. 1, including a different number of atoms according to the width $W$. Each ribbon is separated from its periodic replicas by including a suitable amount of vacuum in the unit cell, both in the out-of-plane and the nonperiodic in-plane directions.

We then use the atomistic simulation toolkit QUANTUMATK $[36,37]$ to calculate the DFT band structure of pristine nanoribbons. For QUANTUMATK band structure calculations, we resorted to an LCAO basis using the SG15 pseudopotential [56] - with the only exception of $\mathrm{BiCl}$ and $\mathrm{SbCl}$ structures, where we use the OPENMX package $[57,58]$. We sample the Brillouin zone of the nanoribbon with a $1 \times 1 \times 16$ Monkhorst-Pack (MP) grid [59] to ensure convergence, while the density mesh cutoff controlling the real-space grid is set to 100 Hartree. We also checked the band structure with GPAW by using a plane-wave basis set with an energy cutoff of $400 \mathrm{eV}$ and an identical MP grid, obtaining an excellent agreement with QUANTUMATK calculations. In both cases, we use the PBE functional with the inclusion of spin-orbit coupling, which is a crucial ingredient here.

To calculate the transmission spectrum at zero bias, we use the NEGF formalism [26] as implemented in QUANTUMATK. We define a transport setup by creating identical left and right electrodes and a central scattering region, as shown in Fig. 2. We create pristine electrodes by using the same material as in the central region, and make sure that electrodes are well screened by repeating the unit cell in the transport direction ( $z$ axis) a suitable amount of times-only once for $\mathrm{BiCl}$ and $\mathrm{SbCl}$, three times for $\mathrm{MoS}_{2}$, and twice for the remaining materials. One or more defects in the central scattering region are created by removing one or more halogen atoms. Note that structures obtained in such a way are not optimized, so that we neglect reconstruction effects. Special care is taken in including a suitable portion of pristine material at both sides of the central scattering region, so that the latter is smoothly connected to the electrodes.

Electrodes for NEGF calculations are sampled with a $1 \times$ $1 \times 150$ MP grid, and we use a slightly lower mesh cutoff of 80 Hartree. Periodic boundary conditions are imposed to the device along the transverse directions $x$ and $y$, while we use Dirichlet boundary conditions for the transport direction $z$.

Finally, magnetic moments are estimated from a Mulliken population analysis [60], where we take the difference between the spin-up and -down populations at each site as the magnitude of the magnetic moment in units of the Bohr magneton.
[1] C. L. Kane and E. J. Mele, $Z_{2}$ Topological Order and the Quantum Spin Hall Effect, Phys. Rev. Lett. 95, 146802 (2005).
[2] C. L. Kane and E. J. Mele, Quantum Spin Hall Effect in Graphene, Phys. Rev. Lett. 95, 226801 (2005). 
[3] F. D. M. Haldane, Nobel lecture: Topological quantum matter, Rev. Mod. Phys. 89, 040502 (2017).

[4] Y. Yao, F. Ye, X.-L. Qi, S.-C. Zhang, and Z. Fang, Spin-orbit gap of graphene: First-principles calculations, Phys. Rev. B 75, 041401(R) (2007).

[5] M. König, S. Wiedmann, C. Brüne, A. Roth, H. Buhmann, L. W. Molenkamp, X.-L. Qi, and S.-C. Zhang, Quantum spin Hall insulator state in HgTe quantum wells, Science 318, 766 (2007).

[6] I. Knez, R.-R. Du, and G. Sullivan, Evidence for Helical Edge Modes in Inverted InAs/GaSb Quantum Wells, Phys. Rev. Lett. 107, 136603 (2011).

[7] F. Reis, G. Li, L. Dudy, M. Bauernfeind, S. Glass, W. Hanke, R. Thomale, J. Schäfer, and R. Claessen, Bismuthene on a SiC substrate: A candidate for a high-temperature quantum spin Hall material, Science 357, 287 (2017).

[8] S. Wu, V. Fatemi, Q. D. Gibson, K. Watanabe, T. Taniguchi, R. J. Cava, and P. Jarillo-Herrero, Observation of the quantum spin Hall effect up to 100 kelvin in a monolayer crystal, Science 359, 76 (2018).

[9] K. Kandrai, G. Kukucska, P. Vancsó, J. Koltai, G. Baranka, Z. E. Horváth, Á. Hoffmann, A. Vymazalová, L. Tapasztó, and P. Nemes-Incze, Evidence for room temperature quantum spin Hall state in the layered mineral jacutingaite, arXiv:1903.02458.

[10] I. Cucchi, A. Marrazzo, E. Cappelli, S. Riccò, F. Y. Bruno, S. Lisi, M. Hoesch, T. K. Kim, C. Cacho, C. Besnard, E. Giannini, N. Marzari, M. Gibertini, F. Baumberger, and A. Tamai, Bulk and Surface Electronic Structure of the DualTopology Semimetal $\mathrm{Pt}_{2} \mathrm{HgSe}_{3}$, Phys. Rev. Lett. 124, 106402 (2020)

[11] C. Wu, B. A. Bernevig, and S.-C. Zhang, Helical Liquid and the Edge of Quantum Spin Hall Systems, Phys. Rev. Lett. 96, 106401 (2006).

[12] C. Xu and J. E. Moore, Stability of the quantum spin Hall effect: Effects of interactions, disorder, and $\mathbb{Z}_{2}$ topology, Phys. Rev. B 73, 045322 (2006).

[13] J. Maciejko, C. Liu, Y. Oreg, X.-L. Qi, C. Wu, and S.-C. Zhang, Kondo Effect in the Helical Edge Liquid of the Quantum Spin Hall State, Phys. Rev. Lett. 102, 256803 (2009).

[14] A. Ström, H. Johannesson, and G. I. Japaridze, Edge Dynamics in a Quantum Spin Hall State: Effects from Rashba Spin-Orbit Interaction, Phys. Rev. Lett. 104, 256804 (2010).

[15] Y. Tanaka, A. Furusaki, and K. A. Matveev, Conductance of a Helical Edge Liquid Coupled to a Magnetic Impurity, Phys. Rev. Lett. 106, 236402 (2011).

[16] J. C. Budich, F. Dolcini, P. Recher, and B. Trauzettel, PhononInduced Backscattering in Helical Edge States, Phys. Rev. Lett. 108, 086602 (2012).

[17] T. L. Schmidt, S. Rachel, F. von Oppen, and L. I. Glazman, Inelastic Electron Backscattering in a Generic Helical Edge Channel, Phys. Rev. Lett. 108, 156402 (2012).

[18] J. I. Väyrynen, M. Goldstein, and L. I. Glazman, Helical Edge Resistance Introduced by Charge Puddles, Phys. Rev. Lett. 110, 216402 (2013).

[19] C.-H. Hsu, P. Stano, J. Klinovaja, and D. Loss, Nuclear-spininduced localization of edge states in two-dimensional topological insulators, Phys. Rev. B 96, 081405(R) (2017).

[20] C.-H. Hsu, P. Stano, J. Klinovaja, and D. Loss, Effects of nuclear spins on the transport properties of the edge of two- dimensional topological insulators, Phys. Rev. B 97, 125432 (2018).

[21] S. Groenendijk, G. Dolcetto, and T. L. Schmidt, Fundamental limits to helical edge conductivity due to spin-phonon scattering, Phys. Rev. B 97, 241406(R) (2018).

[22] J. I. Väyrynen, D. I. Pikulin, and J. Alicea, Noise-Induced Backscattering in a Quantum Spin Hall Edge, Phys. Rev. Lett. 121, 106601 (2018).

[23] P. Novelli, F. Taddei, A. K. Geim, and M. Polini, Failure of Conductance Quantization in Two-Dimensional Topological Insulators due to Nonmagnetic Impurities, Phys. Rev. Lett. 122, 016601 (2019).

[24] N. Mounet, M. Gibertini, P. Schwaller, D. Campi, A. Merkys, A. Marrazzo, T. Sohier, I. E. Castelli, A. Cepellotti, G. Pizzi, and N. Marzari, Two-dimensional materials from highthroughput computational exfoliation of experimentally known compounds, Nat. Nanotechnol. 13, 246 (2018).

[25] S. Haastrup, M. Strange, M. Pandey, T. Deilmann, P. S. Schmidt, N. F. Hinsche, M. N. Gjerding, D. Torelli, P. M. Larsen, A. C. Riis-Jensen, J. Gath, K. W. Jacobsen, J. J. Mortensen, T. Olsen, and K. S. Thygesen, The Computational 2D Materials Database: High-throughput modeling and discovery of atomically thin crystals, 2D Mater. 5, 042002 (2018).

[26] M. Brandbyge, J. L. Mozos, P. Ordejón, J. Taylor, and K. Stokbro, Density-functional method for nonequilibrium electron transport, Phys. Rev. B 65, 165401 (2002).

[27] A. Marrazzo, M. Gibertini, D. Campi, N. Mounet, and N. Marzari, Prediction of a Large-Gap and Switchable Kane-Mele Quantum Spin Hall Insulator, Phys. Rev. Lett. 120, 117701 (2018).

[28] T. Olsen, E. Andersen, T. Okugawa, D. Torelli, T. Deilmann, and K. S. Thygesen, Discovering two-dimensional topological insulators from high-throughput computations, Phys. Rev. Mater. 3, 024005 (2019).

[29] A. Marrazzo, M. Gibertini, D. Campi, N. Mounet, and N. Marzari, Relative abundance of $\mathbb{Z}_{2}$ topological order in exfoliable two-dimensional insulators, Nano Lett. 19, 8431 (2019).

[30] A. Marrazzo, N. Marzari, and M. Gibertini, Emergent dual topology in the three-dimensional Kane-Mele $\mathrm{Pt}_{2} \mathrm{HgSe}_{3}$, Phys. Rev. Research 2, 012063(R) (2020).

[31] Z. Song, C.-C. Liu, J. Yang, J. Han, M. Ye, B. Fu, Y. Yang, Q. Niu, J. Lu, and Y. Yao, Quantum spin Hall insulators and quantum valley Hall insulators of $\mathrm{Bi} X / \mathrm{Sb} X(X=\mathrm{H}, \mathrm{F}, \mathrm{Cl}$ and $\mathrm{Br})$ monolayers with a record bulk band gap, NPG Asia Mater. 6, e147 (2014).

[32] C.-C. Liu, S. Guan, Z. Song, S. A. Yang, J. Yang, and Y. Yao, Low-energy effective Hamiltonian for giant-gap quantum spin Hall insulators in honeycomb $X$-hydride/halide $(X=\mathrm{N}-\mathrm{Bi})$ monolayers, Phys. Rev. B 90, 085431 (2014).

[33] J. O. Sofo, A. S. Chaudhari, and G. D. Barber, Graphane: A two-dimensional hydrocarbon, Phys. Rev. B 75, 153401 (2007).

[34] D. C. Elias, R. R. Nair, T. M. G. Mohiuddin, S. V. Morozov, P. Blake, M. P. Halsall, A. C. Ferrari, D. W. Boukhvalov, M. I. Katsnelson, A. K. Geim, and K. S. Novoselov, Control of graphene's properties by reversible hydrogenation: Evidence for graphane, Science 323, 610 (2009).

[35] See Supplemental Material at http://link.aps.org/supplemental/ 10.1103/PhysRevB.101.155404 for supporting figures and discussion. 
[36] S. Smidstrup, D. Stradi, J. Wellendorff, P. A. Khomyakov, U. G. Vej-Hansen, M.-E. Lee, T. Ghosh, E. Jónsson, H. Jónsson, and K. Stokbro, First-principles Green's-function method for surface calculations: A pseudopotential localized basis set approach, Phys. Rev. B 96, 195309 (2017).

[37] S. Smidstrup, T. Markussen, P. Vancraeyveld, J. Wellendorff, J. Schneider, T. Gunst, B. Verstichel, D. Stradi, P. A. Khomyakov, U. G. Vej-Hansen, M.-E. Lee, S. T. Chill, F. Rasmussen, G. Penazzi, F. Corsetti, A. Ojanperä, K. Jensen, M. L. N. Palsgaard, U. Martinez, A. Blom, M. Brandbyge, and K. Stokbro, QuantumATK: An integrated platform of electronic and atomicscale modeling tools, J. Phys.: Condens. Matter 32, 015901 (2020).

[38] Y. V. Nazarov and Y. M. Blanter, Quantum Transport, Introduction to Nanoscience (Cambridge University Press, Cambridge, 2009).

[39] X. Dang, J. D. Burton, and E. Y. Tsymbal, Magnetic gating of a 2D topological insulator, J. Phys.: Condens. Matter 28, 38LT01 (2016).

[40] J.-H. Zheng and M. A. Cazalilla, Nontrivial interplay of strong disorder and interactions in quantum spin-Hall insulators doped with dilute magnetic impurities, Phys. Rev. B 97, 235402 (2018).

[41] R. R. Nair, I. L. Tsai, M. Sepioni, O. Lehtinen, J. Keinonen, A. V. Krasheninnikov, A. H. Castro Neto, M. I. Katsnelson, A. K. Geim, and I. V. Grigorieva, Dual origin of defect magnetism in graphene and its reversible switching by molecular doping, Nat. Commun. 4, 2010 (2013).

[42] M. A. Khan, M. Erementchouk, J. Hendrickson, and M. N. Leuenberger, Electronic and optical properties of vacancy defects in single-layer transition metal dichalcogenides, Phys. Rev. B 95, 245435 (2017).

[43] X. He, T. He, Z. Wang, and M. Zhao, Neutral vacancy-defectinduced magnetism in SiC monolayer, Physica E 42, 2451 (2010).

[44] W.-F. Li, C. Fang, and M. A. van Huis, Strong spin-orbit splitting and magnetism of point defect states in monolayer $\mathrm{WS}_{2}$, Phys. Rev. B 94, 195425 (2016).

[45] J.-H. Chen, L. Li, W. G. Cullen, E. D. Williams, and M. S. Fuhrer, Tunable Kondo effect in graphene with defects, Nat. Phys. 7, 535 (2011).

[46] S. Azevedo, J. R. Kaschny, C. M. de Castilho, and F. de Brito Mota, Electronic structure of defects in a boron nitride monolayer, Eur. Phys. J. B 67, 507 (2009).

[47] S. Tiwari, M. L. Van de Put, B. Sorée, and W. G. Vandenberghe, Carrier transport in two-dimensional topological insulator nanoribbons in the presence of vacancy defects, 2D Mater. 6, 025011 (2019).

[48] P.-H. Chang, M. S. Bahramy, N. Nagaosa, and B. K. Nikolić, Giant thermoelectric effect in graphene-based topological insu- lators with heavy adatoms and nanopores, Nano Lett. 14, 3779 (2014).

[49] J. Strunz, J. Wiedenmann, C. Fleckenstein, L. Lunczer, W. Beugeling, V. L. Müller, P. Shekhar, N. Traverso Ziani, S. Shamim, J. Kleinlein, H. Buhmann, B. Trauzettel, and L. W. Molenkamp, Interacting topological edge channels, Nat. Phys. 16, 83 (2020).

[50] M. Gibertini, G. Pizzi, and N. Marzari, Engineering polar discontinuities in honeycomb lattices, Nat. Commun. 5, 5157 (2014).

[51] M. Gibertini and N. Marzari, Emergence of one-dimensional wires of free carriers in transition-metal-dichalcogenide nanostructures, Nano Lett. 15, 6229 (2015).

[52] J. P. Perdew, K. Burke, and M. Ernzerhof, Generalized Gradient Approximation Made Simple, Phys. Rev. Lett. 77, 3865 (1996).

[53] J. J. Mortensen, L. B. Hansen, and K. W. Jacobsen, Real-space grid implementation of the projector augmented wave method, Phys. Rev. B 71, 035109 (2005).

[54] J. Enkovaara, C. Rostgaard, J. J. Mortensen, J. Chen, M. Dułak, L. Ferrighi, J. Gavnholt, C. Glinsvad, V. Haikola, H. A. Hansen, H. H. Kristoffersen, M. Kuisma, A. H. Larsen, L. Lehtovaara, M. Ljungberg, O. Lopez-Acevedo, P. G. Moses, J. Ojanen, T. Olsen, V. Petzold, N. A. Romero, J. Stausholm-Møller, M. Strange, G. A. Tritsaris, M. Vanin, M. Walter, B. Hammer, H. Häkkinen, G. K. H. Madsen, R. M. Nieminen, J. K. Nørskov, M. Puska, T. T. Rantala, J. Schiøtz, K. S. Thygesen, and K. W. Jacobsen, Electronic structure calculations with GPAW: a real-space implementation of the projector augmented-wave method, J. Phys. Condens. Matter 22, 253202 (2010).

[55] A. H. Larsen, J. J. Mortensen, J. Blomqvist, I. E. Castelli, R. Christensen, M. Dułak, J. Friis, M. N. Groves, B. Hammer, C. Hargus, E. D. Hermes, P. C. Jennings, P. B. Jensen, J. Kermode, J. R. Kitchin, E. L. Kolsbjerg, J. Kubal, K. Kaasbjerg, S. Lysgaard, J. B. Maronsson, T. Maxson, T. Olsen, L. Pastewka, A. Peterson, C. Rostgaard, J. Schiøtz, O. Schütt, M. Strange, K. S. Thygesen, T. Vegge, L. Vilhelmsen, M. Walter, Z. Zeng, and K. W. Jacobsen, The atomic simulation environmenta Python library for working with atoms, J. Phys. Condens. Matter 29, 273002 (2017).

[56] M. Schlipf and F. Gygi, Optimization algorithm for the generation of ONCV pseudopotentials, Comput. Phys. Commun. 196, 36 (2015).

[57] T. Ozaki, Variationally optimized atomic orbitals for large-scale electronic structures, Phys. Rev. B 67, 155108 (2003).

[58] T. Ozaki and H. Kino, Numerical atomic basis orbitals from $\mathrm{H}$ to Kr, Phys. Rev. B 69, 195113 (2004).

[59] H. J. Monkhorst and J. D. Pack, Special points for Brillouinzone integrations, Phys. Rev. B 13, 5188 (1976).

[60] R. S. Mulliken, Electronic population analysis on LCAO-MO Molecular wave functions. I, J. Chem. Phys. 23, 1833 (1955). 